\title{
ПОВЫШЕНИЕ ТОЧНОСТИ КРАТКОСРОЧНОГО ПРОГНОЗИРОВАНИЯ ЭЛЕКТРОПОТРЕБЛЕНИЯ ГРУПП ТОЧЕК ПОСТАВКИ ЭЛЕКТРОЭНЕРГИИ ГАРАНТИРУЮЩИХ ПОСТАВЩИКОВ ВТОРОГО УРОВНЯ
}

\author{
Хомутов Станислав Олегович1, \\ homutov.so@yandex.ru
}

\author{
Сташко Василий Иванович 1 , \\ altgtu@mail.ru
}

\section{Серебряков Николай Александрович1, na_serebryakov@altke.ru}
1 Алтайский государственный технический университет им. И.И. Ползунова, Россия, 656038, г. Барнаул, пр. Ленина, 46.

\begin{abstract}
Актуальность обусловлена необходимостью повышения точности краткосрочного прогнозирования электропотребления групп точек поставки электроэнергии гарантирующих поставщиков второго уровня. Результаты данного прогноза используются системным оператором при формировании диспетчерского графика выработки и потребления электроэнергии. Ошибки прогнозирования, как правило, приводят к увеличению расхода первичных энергоресурсов на выработку электроэнергии за счет необоснованных пусков и остановов генерирующего оборудования, а также к увеличению потерь при передаче электроэнергии, вследствие выбора неоптимальной схемы электрических сетей. Так как потребление электроэнергии зависит от множества фракторов, задача краткосрочного прогнозирования данного временного ряда является слабоформализуемой. В данных условиях традиционные средства математической статистики и имитационного моделирования не позволяют строить адекватнье прогнозные модели. До недавнего времени единственным адекватным методом прогнозирования потребления электроэнергии был метод экспертных оценок. В настоящее время для краткосрочного прогнозирования электропотребления энергосистемы региона страны или группы точек поставки электроэнергии гарантирующего поставщика первого уровня все больщее применение находят инструменты нейронных сетей. Однако разработанные модели мало пригодны для прогнозирования почасового потребления электрической энергии групп точек поставки электроэнергии гарантирующих поставщиков второго уровня. Краткосрочное прогнозирование потребления электроэнергии данных объектов осложнено тем, что, помимо стандартных временных и метеорологических фракторов, необходимо учитывать надежность электросетевого оборудования 6-110 кB, режим работы потребителей электрической энергии с мощностью 670-10000 кBm, а также наличие центрального отопления и горячего (холодного) водоснабжения в населенном пункте, питающемся от группы точек поставки электроэнергии. Для них остается открытым вопрос выбора оптимальной архитектуры и конфигурации нейросетевой модели, а также алгоритма ее обучения, при использовании которых достигается требуемая точность прогнозирования.

Цель: повышение точности краткосрочного прогнозирования электропотребления групn точек поставки электроэнергии гарантирующего поставщика второго уровня с помощью инструментов нейронных сетей и глубокого машинного обучения.

Методы: методы корреляционного и фракторного анализа, теории искусственных нейронных сетей и машинного обучения. Программная реализация теоретических выкладок выполнена с помощью библиотеки глубокого машинного обучения Tensor flow Keras на языке программирования Python 3.6.

Результаты. Разработан нейросетевой алгоритм краткосрочного прогнозирования электропотребления групп точек поставки электроэнергии гарантирующего поставщика второго уровня с адаптивными в процессе обучения параметрами скорости обучения и момента инерции. Выполнена программная реализация данного алгоритма в библиотеке глубокого машинного обучения Tensor flow Keras. Использование данной искусственной нейронной сети позволило снизить среднемесячную относительную ошибку прогнозирования на 5,14 \%.
\end{abstract}

\section{Ключевые слова:}

Краткосрочное прогнозирования электропотребления, искусственные нейронные сети, алгоритм обучения, оптовый рынок электроэнергии и мощности, группа точек поставки электроэнергии, градиентный спуск.

\section{Введение}

В настоящее время на выработку электроэнергии потребляется огромное количество первичных энергоресурсов, таких как уголь, нефть, газ. Немалая часть из них расходуется на пуски генерирующего оборудования. Для минимизации необоснованных пусков генерирующего оборудования формируется диспетчерский график производства и потребления электроэнергии. Системный оператор использует результаты краткосрочного прогнозирования электропотребления (short-term load forecasting - STLF) крупных потребителей электроэнергии при формиро- вании диспетчерского графика. То есть точность STLF влияет на режим функционирования электроэнергетических систем. Ошибки прогнозирования, как правило, приводят к необоснованным пускам и остановам генерирующего оборудования, а также к выбору неоптимальной схемы электрических сетей. Это, в свою очередь, приводит к снижению эффективности функционирования энергосистемы, за счёт увеличения расхода первичных энергоресурсов на выработку электрической энергии, а также к увеличению потерь при ее передаче. Заблаговременная оценка надежности является необходимым условием для 
функционирования и развития современных электроэнергетических систем (ЭЭС), которые характеризуются развитием распределенной генерации, возобновляемых источников энергии, интеллектуализацией, что усложняет ЭЭС и вносит свои коррективы в процесс оценки надежности ЭЭС [1]. Кроме того, растущая доступность возобновляемых источников энергии увеличивает нестабильность баланса мощности энергосистемы, поскольку появляется дополнительная неопределенность на стороне производства электроэнергии [2, 3]. Совокупность данных факторов делают краткосрочное прогнозирование электропотребления критическим аспектом обеспечения надежности и эффективности энергосистемы [2].

Ценовая конъюнктура на оптовом рынке электроэнергии и мощности (ОРЭМ) складывается так, чтобы стимулировать крупных покупателей электроэнергии, таких как гарантирующие поставщики электроэнергии (ГП), придерживаться собственных прогнозов. Для достижения адекватных финансовых результатов ГП необходимо прогнозировать собственное почасовое потребление электроэнергии по всем группам точек поставки электроэнергии (ГТП) на ОРЭМ с высокой точностью. Достижение высокой точности прогнозирования затруднено наличием множества факторов, влияющих на электрическую нагрузку [4].

На сегодняшний день энергосбытовыми компаниями в основном используются интуитивные методы прогнозирования электропотребления. Вследствие человеческого фактора и ряда других причин использование данных методов не позволяет уменьшить ошибку прогноза ниже определенного значения. Наиболее эффективным путем повышения качества STLF является формализация данного процесса. Однако наличие множества факторов, влияющих на потребление электроэнергии, а также стохастический характер некоторых из них делает задачу STLF слабоформализуемой. В условиях неопределенности традиционные методы математической статистики или имитационного моделирования не позволяют строить адекватные модели объектов [5]. В настоящее время стремительно развиваются методы искусственного интеллекта и глубокого машинного обучения. Данные методы являются эффективным инструментом решения слабоформализуемых или неформализуемых задач. Несмотря на то, что теоретическая основа для применения нейросетевых алгоритмов была разработана еще во второй половине XX в., до недавнего времени применение инструментов машинного обучения для решения задачи STLF сдерживалось недостатком вычислительной мощности ЭВМ.

За последние несколько десятилетий ученые разработали множество методов повышения точности STLF, которые можно разделить на: методы, основанные на математической статистике, методы искусственного интеллекта и гибридные методы $[2,4]$. Невзирая на большое количество существующих алгоритмов краткосрочного прогнозирования электропотребления, ни один из них нельзя назвать универсальным. Разработанные методы предназначены либо для прогнозирования электрической нагрузки энерго- системы региона страны или ГТП гарантирующего поставщика первого уровня [6], либо для прогнозирования электропотребления конечных потребителей, таких как здания или промышленные предприятия [7-10]. Однако данные модели непригодны для прогнозирования почасового потребления электрической энергии ГТП гарантирующих поставщиков второго уровня. Краткосрочное прогнозирование потребления электроэнергии данных объектов осложнено, помимо стандартных временных и метеорологических факторов, надежностью электросетевого оборудования 6-110 кВ, режимом работы потребителей электрической энергии с мощностью 670-10000 кВт, а также наличием центрального отопления и горячего (холодного) водоснабжения в населенных пунктах, относящихся к ГТП.

C учетом дополнительных факторов при STLF остаются открытыми вопросы выбора оптимальной архитектуры и конфигурации нейросетевой модели, при которых достигается максимальная точность прогнозирования. В то же время до сих пор не существует формализованных методов проектирования и настройки параметров искусственных нейронных сетей (ИНС). Под каждую конкретную задачу необходимо разрабатывать собственную нейронную сеть, для которой оптимальное значение всех гиперпараметров подбирается на основе многочисленных опытов с обучающей и тестовой выборкой.

Так как гарантирующие поставщики второго уровня имеют до нескольких десятков групп точек поставки электроэнергии на ОРЭМ, для каждой из которых ежедневно необходимо составить прогноз электропотребления на следующие сутки, для них особое значение приобретает скорость обучения прогнозной модели на свежих статистических данных. Для данной категории объектов прогнозирования необходимо, по возможности, выбирать модели не слишком требовательные к вычислительным мощностям.

Стоит также отметить, что точность краткосрочного прогнозирования зависит не только от математической модели, но также от набора связанных статистических данных [10]. В случае, если исходные данные для прогнозирования сильно зашумлены, или учтены не все влияющие факторы, результаты прогнозирования будут неудовлетворительными. Следовательно, формирование обучающей выборки на основе статистических данных, наряду с проектированием прогнозной модели, является важнейшей составляющей STLF.

\section{Анализ основных факторов, влияющих}

на потребление электроэнергии ГТП ГП второго уровня

Слово «прогноз» в буквальном переводе с греческого означает «перед знанием», т. е. до того, как появится реальное знание (наблюдение) предмета или явления, до того, как событие наступит [11]. Прогнозирование электропотребления позволяет инфраструктурным организациям моделировать и прогнозировать нагрузки для поддержания баланса между производством и спросом, снижать себестоимость продукции, оценивать реальную цену на электроэнергию, а также управлять режимом и планировать вве- 
дение будущих мощностей. В литературе хорошо освещен вопрос краткосрочного прогнозирования спроса на электроэнергию. Данный вид прогнозирования важен при управлении энергосистемой, пусках энергоблоков, экономической диспетчеризации, а также на рынках электроэнергии [7].

Предварительным этапом прогнозирования любого временного ряда является анализ факторов, влияющих на поведение данной функциональной зависимости. В случае краткосрочного прогнозирования временного ряда электропотребления ГТП гарантирующего поставщика второго уровня с помощью инструментов ИНС данный анализ приобретает особое значение. Выбор высококоррелированных входных данных имеет ключевое значение для моделей прогнозирования потребления электроэнергии, построенных на основе искусственного интеллекта [8]. К ним можно отнести временные факторы, метеорологические факторы, факторы надежности питающей сети и режима работы крупных потребителей электроэнергии ГТП, а также факторы наличия центрального отопления и горячего (холодного) водоснабжения в населенных пунктах, относящихся к ГТП. Детальное понимание факторов, влияющих на спрос на электроэнергию, может помочь смягчить последствия от нестабильности производства электроэнергии за счёт тарифного стимулирования потребления электроэнергии в определенные часы суток [3].

\section{Анализ влияния временных факторов на поведение} временного ряда потребления электроэнергии ГТП

Временные факторы обусловлены наличием циклических закономерностей в поведении временного ряда электрической нагрузки ГТП ГП различной длительности (сутки, неделя, год). Данная группа факторов обуславливает основные тенденции изменения суточного графика электрической нагрузки (СГЭН) в течение определенного интервала времени. Знание динамики поведения временного ряда в ту или иную фазу цикла позволяет производить более качественную кластеризацию исходных данных при STLF. Целью кластеризации является разбиение исходных данных в однородные подмножества, называемые кластерами. Однородность измеряется в соответствии с различными критериями, такими как внутриклассовые и межклассовые отклонения или меры различия [12].

При анализе суточных изменений графика электрической нагрузки ГТП гарантирующего поставщика второго уровня можно отметить, что имеет ярко выраженные утренний и вечерний пики, между которыми располагается зона относительной просадки электропотребления, а также значительное снижение потребления электроэнергии в ночные часы суток. Эти характерные особенности СГЭН обусловлены повседневным ритмом жизни людей, режимом работы предприятий и т. д.

При рассмотрении недельного цикла можно утверждать, что форма графиков электропотребления сильно закоррелирована с повседневным образом жизни людей и режимом работы предприятий. В течение рабочей недели форма СГЭН практически не изменяется.
Небольшие отличия имеют СГЭН в начале и конце рабочей недели. Значительное изменение формы СГЭН происходит в выходные дни вследствие остановки некоторой части оборудования промышленных предприятий, отсутствия необходимости подготовки к следующему рабочему дню у части населения.

В течение года суточные графики потребления электроэнергии ГТП ГП постоянно изменяют свою форму. Это обусловлено постоянным изменением температуры воздуха, длительности светового времени суток, сезонными работами и т. д. В целом тенденции изменения суточного потребления электроэнергии повторяются из года в год. При рассмотрении цикла длительностью в один год необходимо учитывать, что праздничные дни характеризуются значительной просадкой электропотребления, а также ростом электрической нагрузки в дневные часы суток, предшествующим празднованию. Учет признаков праздничных и предпраздничных дней позволяет избежать значительных отклонений фактического потребления электроэнергии от прогнозного в период праздничных суток.

\section{Влияние метеорологических факторов на временной ряд электрической нагрузки ГТП гарантирующего поставщика}

Помимо внутренних тенденций поведение временного ряда потребления электроэнергии в краткосрочной перспективе определяется группой метеорологических факторов. Погодные условия являются наиболее влиятельными экзогенными переменными при краткосрочном прогнозировании электрической нагрузки [13].

На основании схожих исследований [14] можно сделать вывод, что между временными рядами потребления электроэнергии и температуры воздуха имеется сильная отрицательная корреляционная связь. Отрицательная корреляция сохраняется до наступления периода экстремально высоких температур. При среднесуточной температуре воздуха выше комфортных $20{ }^{\circ} \mathrm{C}$ появляется необходимость включения дополнительного климатического оборудования, которое увеличивает потребление электроэнергии. На рис. 1 представлены суточные графики электрической нагрузки группы точек поставки электроэнергии «Кулундинская» в период 20-24 марта 2017.

В период 20-24 марта 2017 произошло резкое повышение температуры в городе Славгороде с $-13,3{ }^{\circ} \mathrm{C}$ 20.03.2017 до $-2{ }^{\circ} \mathrm{C} 23.03 .2017$ и 24.03.2017. Графики на рис. 1 хорошо иллюстрируют сильную зависимость почасовых объемов электропотребления ГТП ГП второго уровня от температуры наружного воздуха. Также стоит отметить, что изменение объемов потребления электроэнергии, вызванное изменением температуры, имеет некоторую инерционность. Данное явление обусловлено тем, что изменение температуры в отапливаемых помещений запаздывает по отношению к изменениям температуры наружного воздуха. Предлагается учитывать инерционность при STLF с помощью фактора дисперсии выборки из 24-х предыдущих значений температуры: 


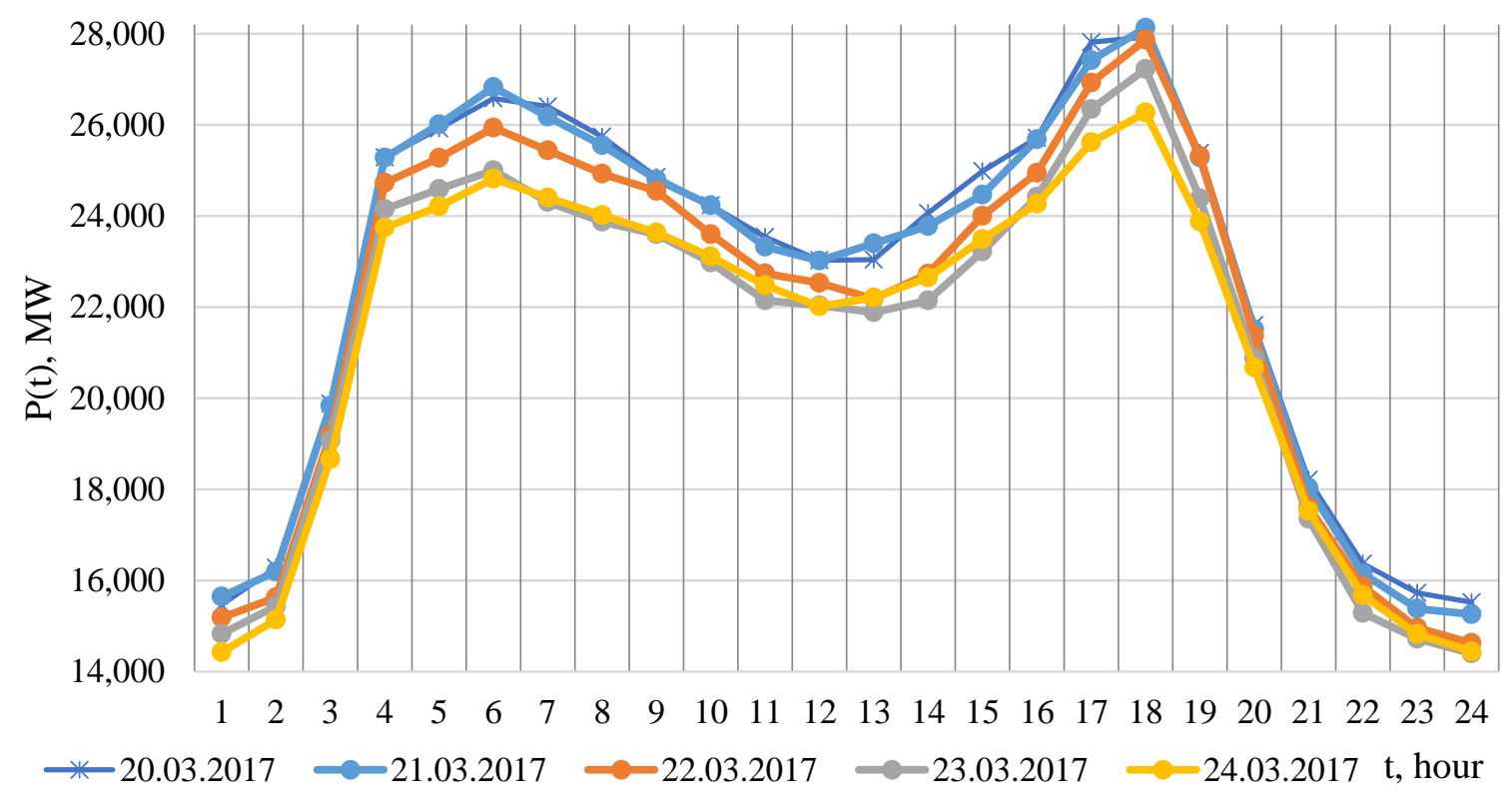

Рис. 1. Суточные графики электрической нагрузки группы точек поставки электроэнергии «Кулундинская» в период 20-24 марта 2017

Fig. 1. Daily load curve of delivery point cluster «Kulundinskaya» in the period 20-24 March 2017

$$
D=\frac{1}{24} \sum_{i=1}^{24}\left(t_{i}-\bar{t}\right)^{2}
$$

где $D$ - выборочная дисперсия последних 24-х значений температуры воздуха; $t_{i}$ - температура воздуха в час $i ; \bar{t}$ - выборочное среднее.

Рост дисперсии $D$ из выражения (1) будет сигнализировать о серьезных изменениях температурного режима. Так как дисперсия рассчитывается на основании выборки из 24-х значений, кратковременные перепады температуры наружного воздуха не будут вызывать ее значительного роста.

Влияние ветровой нагрузки на потребление электроэнергии ГТП невозможно оценить однозначно. В работе [9] говорится, что использование скорости ветра как отдельного фактора при STLF снижает точность прогнозирования. Однако однозначно можно говорить о том, что ветер влияет на субъективное ощущение человеком температуры наружного воздуха, а также увеличивает теплопотери в отапливаемых помещениях. Величины температуры наружного воздуха и скорости ветра можно одновременно учесть с помощью ветро-холодового индекса Стедмана (The Steadman Wind Chill) [15]:

$$
T_{W C}=1,41-1,162 \mathrm{~V}+0,98 T+0,0124 V^{2}+0,0185 T V \text {, }
$$

где $T_{W C}-$ ветро-холодовой индекс; $V$ - скорость ветра, м/c; $T$ - температура воздуха, ${ }^{\circ} \mathrm{C}$.

Ветро-холодовой индекс представляет собой регрессионную модель второго порядка, полученную на основании статистических данных о биометрических измерениях, производившихся во многих странах с 1940 по 1994 гг. Использование ветро-холодового индекса при STLF позволяет уменьшить размерность факторного пространства без потери полезной информации, описывающей поведение временного ряда потребления электроэнергии ГТП.
Следующим по степени влияния на электрическую нагрузку метеорологическим фактором является естественная освещенность. Значительное снижение естественной освещенности происходит только при наличии дождевых облаков нижнего яруса. Также нельзя говорить, что уменьшение освещенности неизменно влечет увеличение электропотребления ГТП. В летнее время года снижение освещенности сопровождается снижением электрической нагрузки. Данное обстоятельство объясняется тем, что в дождливую погоду отключается часть климатического оборудования, а также отпадает необходимость полива сельскохозяйственных культур. На основании вышеизложенного, а также в связи с проблематичностью получения статистических данных о естественной освещенности по всем районам из зоны деятельности гарантирующего поставщика можно сделать вывод о целесообразности применения фактора наличия осадков при STLF.

Учет влияния остальных метеорологических факторов необоснованно увеличит размерность факторного пространства, при том что увеличения точности прогнозирования это не повлечет.

\section{Учёт случайных воздействий \\ на временной ряд потребления электроэнергии}

Не все данные, необходимые для составления прогноза потребления электроэнергии, являются детерминированными. К стохастическим факторам, которые оказывают влияние на результаты STLF, можно отнести аварийные отключения на электросетевом оборудовании, а также поломки основного оборудования крупных потребителей электроэнергии ГТП гарантирующего поставщика второго уровня. Темой отдельного исследования является прогнозирование отказов элементов электросетевого оборудования с помощью аппарата нейронных сетей [16]. 
Однако существуют данные, необходимые для составления STLF, которые не являются детерминированными, но в то же время вероятность их возникновения достаточно велика. К ним можно отнести информацию о плановых ремонтных работах на электросетевом оборудовании, данные о режиме работы крупных потребителей электроэнергии ГТП ГП, данные о начале и конце отопительного периода, данные об отключениях горячего и холодного водоснабжения Данные факторы создают дополнительную неопределенность при составлении прогноза. Однако без их учета резко снижается точность STLF.

АО «Алтайкрайэнерго» является гарантирующим поставщиком электроэнергии второго уровня на части территории Алтайского края. В литературе хорошо освещен вопрос разработки моделей краткосрочного прогнозирования электропотребления энергосистемы субъекта РФ [6]. При краткосрочном прогнозировании электрической нагрузки таких крупных объектов можно не учитывать ремонтные работы на сетевом оборудовании ниже 220 кВ, а также режим работы большинства потребителей электроэнергии. В то же время отключения на сетевом оборудовании 6110 кВ режим работы потребителей электроэнергии мощностью свыше 670 кВ в значительной степени сказывается на потреблении электроэнергии ГТП ГП второго уровня. На рис. 2 представлены суточные графики потребления электроэнергии ГТП «Корчинская» при плановых ремонтах на сетевом оборудовании филиала ПАО «МРСК Сибири» - «Алтайэнерго».

26.04.2017 на подстанциях «Романовская» и «Мамонтовская», входящих в ГТП «Корчинская», проводились плановые работы по чистке изоляции. Как мы видим из рис. 2, потребление электроэнергии ГТП «Корчинская» в часы ремонтных работ уменьшилось в среднем на 69,77 \%, а после подачи напряжения произошел скачок электрической нагрузки. Во избе- жание больших отклонений фактического потребления электроэнергии от прогнозного необходимо учитывать все плановые работы на сетевом оборудовании, обеспечивающем транспорт электрической энергии до конечного потребителя.

При прогнозировании почасовых объемов электропотребления ГТП гарантирующих поставщиков второго уровня необходимо учитывать режим работы крупных потребителей электроэнергии с мощностью свыше 670 кВт, так как электрическая нагрузка данных потребителей соизмерима с нагрузкой всей ГТП. Учет режима работы более мелких потребителей электроэнергии ГТП ГП значительно усложнит архитектуру модели прогнозирования без увеличения точности STLF.

Таким образом, к основным факторам, оказывающими решающее воздействие на поведение временного ряда электропотребления ГТП ГП второго уровня в краткосрочной перспективе, можно отнести:

- порядковый номер дня в года;

- порядковый номер дня в неделе;

- длина светового дня;

- признак предпраздничного дня;

- признак праздничного дня;

- признак каникул в образовательных учреждениях;

- признак наличия центрального отопления;

- признак наличия горячего водоснабжения;

- признак наличия холодного водоснабжения;

- почасовые значения ветро-холодового индекса, ${ }^{\circ} \mathrm{C}$;

- дисперсия 24-х предыдущих значений температуры наружного воздуха, ${ }^{\circ} \mathrm{C}$;

- количество осадков, мм;

- режим работы всех потребителей электроэнергии ГТП с мощностью свыше 670 кВт;

- режим работы всех фидеров, питающих потребителей электроэнергии ГТП

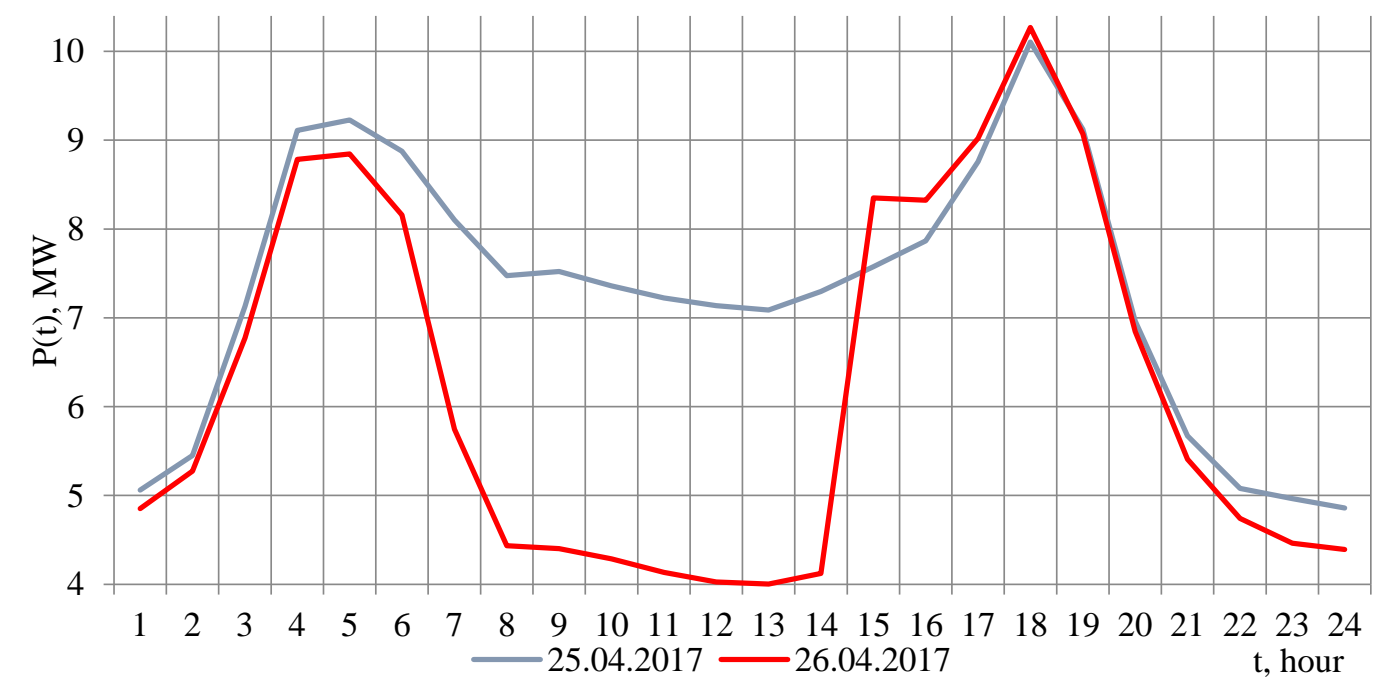

Pис. 2. Суточные графики потребления электроэнергии группы точек поставки «Корчинская» при плановых ремонтных на сетевом оборудовании филиала ПАО «МРСК Сибири» - «Алтайэнерго»

Fig. 2. Daily load curve of delivery point cluster «Korchinskaya» with scheduled repairs on electric grid equipment of the branch of PJSC «IDGC of Siberia» - «Altaienergo» 
На основании набора факторов формируется выборка, предназначенная для обучения и тестирования ИНС. Из множества параметров, определяющих электрическую нагрузку, выбираются те, которые могут быть внесены в обучающую выборку, то есть доступные в открытых источниках или вычисляемые на основании открытой информации [6].

\section{Проектирование искусственной нейронной сети для решения задачи прогнозирования электропотребления ГТП «на сутки вперед»}

STLF является сложной задачей. В настоящее время для решения подобных задач используется аппарат искусственных нейронных сетей. Способность к обобщению информации, описывающей нелинейные зависимости в сложных объектах, процессах и явлениях, неотъемлемая черта ИНС, присущая ей по внутренней природе - по структуре и по способу функционирования на основе обучения [17].

Проектирование ИНС начинается с выбора парадигмы обучения. Для решения задачи STLF ГTП ГП второго уровня хорошо подходит парадигма обучения с учителем, так как при возможности формирования желаемого отклика из данных тренировочной выборки ИНС, обучаемые с учителем, достигают более высокой точности прогноза по сравнению с нейронными сетями, обучаемыми на основе самоорганизации.

В процессе обучения ИНС с учителем все параметры в модели, управляемой данными (Data-driven models), изменяются на основании систематического сравнения отклика модели со статистическими данными [18]. То есть целью обучения является получение отклика ИНС наиболее статистически схожего с желаемым:

$$
\left\{\begin{array}{l}
e(n)=d(n)-y(n) ; \\
E(n)=\frac{1}{2} e^{2}(n),
\end{array}\right.
$$

где $e(n)$ - абсолютная ошибка ИНС на шаге обучения $n ; d(n)$ - желаемый отклик ИНС; $y(n)$ - фактический отклик сети ИНС; $E(n)$ - функция потерь.

Только после того, как выходная ошибка сети становится ниже требуемого значения, модель может быть пригодна для практического применения со свежими входными данными [19].

На следующем этапе выбирается архитектура нейронной сети, которая связана с алгоритмом обучения. Методы глубокого обучения широко и успешно применяются для ряда задач, таких как классификация и распознавание объектов [20]. В отличие от обычных структур ИНС, глубокая нейронная сеть (DNN) имеет больше одного скрытого слоя. Многослойная структура вычислительных слоев увеличивает способность сети к абстрагированию признаков, что делает их более эффективными в изучении сложных нелинейных моделей [7].

К ИНС, построенным на методах глубокого машинного обучения, можно отнести сети прямого распространения, такие как многослойные персептроны и сверточные нейронные сети, рекуррентные нейронные сети [7] и скрытые Марковские сети. Многосту- пенчатые системы, связанные с цепями Маркова, описывают широкий спектр механических, физических и экономических процессов [21]. Для прогнозирования состояния таких систем хорошо подходят сети Маркова [19]. Ограничением применения рекурентных нейронных сетей и скрытых сетей Маркова для решения задачи STLF ГTП гарантирующего поставщика второго уровня являются большие затраты вычислительных мощностей на обучение данных сетей. Это, в свою очередь, вызывает значительное увеличение времени обучения ИНС. Так как ГП второго уровня могут иметь более десяти ГТП на ОРЭМ (в частности, АО «Алтайкрайэнерго» имеет 15 ГТП), по которым ежедневно формируется прогноз электропотребления «на сутки вперед», скорость обучения прогнозной модели является критичным параметром.

При решении схожих с STLF задач - распознавании рукописного текста и объектов на изображении, хорошо зарекомендовали себя сети прямого распространения. Ограничением применения сверточных ИНС является сложность представления входных данных, так как сверточные слои оперируют с двух- и трехмерными массивами данных, в то время как обучающая выборка представляет собой одномерный вектор-столбец. На основании вышеизложенного можно сделать вывод о том, что многослойный персептрон является оптимальной архитектурой ИНС для решения задачи краткосрочного прогнозирования почасового потребления электроэнергии ГТП гарантирующего поставщика второго уровня. Обучение многослойных персептронов происходит на основании алгоритма обратного распространения ошибки.

На рис. 3 представлена структурная схема многослойного персептрона.

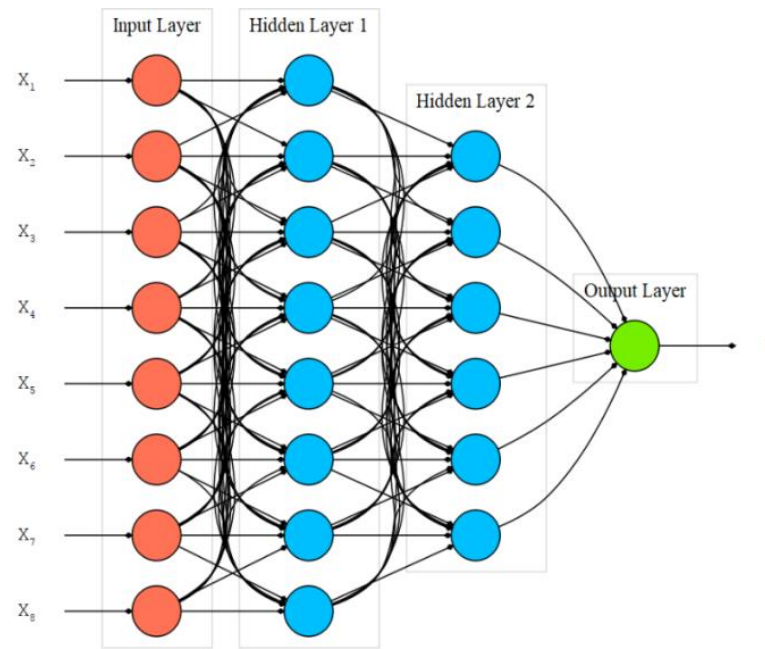

Рис. 3. Структурная схема многослойного персептрона

Fig. 3. Structural diagram of a multilayer perceptron

В рассматриваемой структуре нейроны регулярным образом организованы в слои. Слой содержит совокупность нейронов с едиными входными сигналами [22]. Нейронная сеть, изображенная на рис. 3, состоит из: входного слоя сенсорных элементов, нескольких скрытых слоев, в которых происходят ос- 
новные вычисления в ИНС, и выходного слоя, формирующего итоговый отклик ИНС на входное воздействие.

\section{Выбор оптимального значения гиперпараметров ИНС}

Нейронные сети прямого распространения характеризуются набором гиперпараметров: значение момента инерции и скорости обучения ИНС на каждой итерации обучающего цикла, количество скрытых слоев, а также нейронов в каждом скрытом слое, наличие смещения функции активации. Выбор оптимального значения гиперпараметров ИНС напрямую влияет на способность ИНС адекватно описывать поведение временного ряда почасовых объемов электропотребления ГТП. В настоящее время не существует формализованных методов определения оптимального значения гиперпараметров. Преодоление вышеприведенных недостатков на практике достигается перебором различных вариантов архитектур, типов нейронов, начальных условий, методов обучения и дальнейшим выбором наиболее удачного решения [23].

Количество нейронов во входном и выходном слоях определяется на основании размера векторов входных данных и желаемого отклика ИНС:

$$
\left\{\begin{array}{l}
m_{0}=24 \cdot f \\
m_{3}=24
\end{array},\right.
$$

где $m_{0}$ - размер входного сенсорного слоя ИНС; $24 \cdot f$ суточные значения величин основных влияющих факторов; $m_{3}$ - размер выходного слоя, соответствующий 24-м значениям прогнозного СГЭН на следующие сутки.

Размер и количество скрытых слоев напрямую влияет на ассоциативную память нейронной сети. Очень важно определить оптимальное количество нейронов в каждом скрытом слое, так как малое количество нейронов не позволит ИНС извлечь всю полезную информацию из данных обучающей выборки, а сеть со слишком большим количеством нейронов в скрытых слоях будет склонна к переобучению. В настоящее время существуют только практические рекомендации по выбору оптимального размера скрытых слоев [24]:

$$
\left\{\begin{array}{l}
m_{1}=m_{3} \cdot\left(\sqrt[3]{\frac{m_{0}}{m_{3}}}\right)^{2} \\
m_{2}=m_{3} \cdot \sqrt[3]{\frac{m_{0}}{m_{3}}}
\end{array} ;\right.
$$

где $m_{1}-$ количество нейронов в первом скрытом слое; $m_{2}$ - количество нейронов во втором скрытом слое.

Однако оптимальный размер скрытых слоев определяется исключительно на основании многочисленных экспериментов с обучающей и тестовой выборкой.

Остальные гиперпараметры ИНС вытекают из принципа функционирования искусственного нейрона и алгоритма обучения ИНС. Искусственный нейрон - это математическая модель, имитирующая биологический нейрон центральной нервной системы, который имеет нелинейную реакцию на входные сиг- налы [25]. Математическим описанием функционирования является выражение:

$$
\left\{\begin{array}{l}
u_{k}=\sum_{j=1}^{m} w_{k j} x_{j} \\
v_{k}=u_{k}+b_{k}, \\
y_{k}=\varphi\left(v_{k}\right)
\end{array}\right.
$$

где $u_{k}-$ взвешенная сумма входных воздействий на нейрон $k ; w_{k j}-$ вес синаптической связи, соединяющей нейрон $k$ и нейрон $j ; x_{j}-$ входной сигнал нейрона $k$ от нейрона $j ; v_{k}-$ индуцированное локальное поле нейрона $k ; b_{k}-$ порог активации нейрона $k ; y_{k}$ - отклик на входные воздействия нейрона $k ; \varphi\left(v_{k}\right)$ - функция активации.

Алгоритм обратного распространения ошибки предполагает прямой и обратный проходы вычислений. При прямом проходе веса синаптических связей не изменяются, а функциональный сигнал, передающийся от слоя к слою, формирует итоговый отклик сети на основании выражения (6). При обратном проходе вычисляется функция потерь для каждого нейрона скрытых и выгодного слоев на основании выражения (3). Вычисляется сумма энергий ошибки по всем нейронам выходного слоя:

$$
E_{C}(n)=\frac{1}{2} \sum_{j \in C} e_{j}^{2}(n),
$$

где $C$ - множество нейронов выходного слоя.

Из выражений (3), (6), (7) видно, что функция энергии ошибки зависит только от свободных параметров ИНС (весов синаптических связей и порогов активации). Обучение сети заключается в определении составляющих вектора весов ее синаптических связей, доставляющих минимум суммарной квадратичной ошибки сети (7) [26]. Геометрической интерпретацией функции потерь является поверхность ошибки в пространстве свободных параметров ИНС. То есть в процессе обучения необходимо произвести итеративную коррекцию свободных параметров ИНС с целью нахождения глобального минимума на поверхности ошибки.

Последовательная выпуклая оптимизация играет ключевую роль в решении задач, где постоянно обновляется статистическая информация [27]. То есть имеется целевая функция, для которой необходимо найти максимум или минимум за счёт оптимизации некоторых ее скалярных параметров. Если целевая функция дифференцируема по своим параметрам, то градиентный спуск является относительно эффективным методом оптимизации [28]. Поиск глобального минимума на поверхности ошибки с помощью метода градиентного спуска (SGD) происходит на основании цепного правила:

$$
\begin{gathered}
\frac{\partial E(n)}{\partial w_{k j}(n)}=\frac{\partial E(n)}{\partial e_{k}(n)} \frac{\partial e_{k}(n)}{\partial y_{k}(n)} \frac{\partial y_{k}(n)}{\partial v_{k}(n)} \frac{\partial v_{k}(n)}{\partial w_{k j}(n)}= \\
=-e_{k}(n) \varphi^{\prime}\left(v_{k}(n)\right) x_{j}(n)=-\delta_{k}(n) x_{j}(n),
\end{gathered}
$$


где $\delta_{k}(n)$ - локальный градиент нейрона $k$ на шаге обучающего цикла $n$.

Исходя из выражения (8), в случае использования сигмоидальной функции активации, коррекция веса синаптической связи принимает вид:

$$
\Delta w_{k j}(n)=\eta \delta_{k}(n) x_{j}(n)+\alpha \Delta w_{k j}(n-1),
$$

где $\Delta w_{k j}(n)$ - корректировка веса взаимодействия между нейронами $k$ и $j$ на шаге обучения $n ; \eta$ - параметр скорости обучения; $\alpha$ - параметр момента инерции; $\Delta w_{k j}(n-1)$ - корректировка веса взаимодействия между нейронами $k$ и $j$ на шаге обучения $n-1$.

На основании выражения (9) можно сделать вывод, что параметры скорости обучения и момента инерции на каждой итерации обучающего цикла влияют на способность алгоритма к схождению к глобальному минимуму на поверхности ошибки, в области которого функция потерь (7) принимает минимальное значение.

Алгоритм обратного распространения ошибки, с помощью которого обучается ИНС, при всех его достоинствах, связанных со скоростью работы, простотой и т. д., имеет существенный недостаток, заключающийся в том, что при обучении ИНС за счет минимизации ошибки методом градиентного спуска можно получить не абсолютное (глобальное) значение минимума, а локальное (т. е. данная нейронная сеть будет недоучена). Современные алгоритмы обучения ИНС, такие как ADAM [28], позволяют преодолеть данный недостаток за счёт применения адаптивных параметров скорости обучения и постоянной момента инерции. ADAM является алгоритмом оптимизации первого порядка, основанным на градиентном спуске. Он эффективен в вычислительном отношении и подходит для оптимизации моделей с большим набором параметров [29]. Адаптивные методы обучения позволяют осуществить градиентный спуск к глобальному минимуму практически при любом «ландшафте» поверхности ошибки за счёт эффективного прохождения локальных минимумов и «плоских» участков на поверхности ошибки.

\section{Способы усиления алгоритма обучения и борьбы с переобучением ИНС}

Глубокие нейронные сети с большим количеством свободных параметров являются очень мощными системами машинного обучения. Однако в таких сетях переобучение является серьезной проблемой [12]. Переобучение можно определить по кривой обучения (learning curve), которая описывает частоту появления ошибок в зависимости от количества эпох. Переобучение соответствует точке, в которой ошибка тестирования начинает увеличиваться, в то время как ошибка обучения продолжает уменьшаться. Это означает, что модель запоминает данные из обучающей выборки, но не может предсказать поведение объекта. Таким образом, излишнее обучение со сложной моделью прогнозирования приводит к ухудшению обобщающей способности сети. Существует несколько методов предотвращения переобучения, таких как отключение случайных нейронов скрытых слоев (Dropout) или ранняя остановка (early stopping) [23, 30].
Для контроля обобщающей способности ИНС необходимо производить перекрестную проверку сети (cross-validation) после каждой итерации обучающего цикла. Для этого статистические данные некоторым образом разделяются на обучающую и тестовую выборки. Персептрон обучается на данных из обучающей выборки, а обобщающая способность оценивается с помощью тестового множества, образы из которого неизвестны сети в течение цикла обучения.

Эффективность ранней остановки алгоритма обучения (early stopping) определяется тем, что исходное значение весов синаптических связей и порогов активации ИНС имеет случайное распределение по нормальному закону. То есть невозможно заранее определить «ландшафт» поверхности ошибки и количество эпох обучающего цикла, за которые рабочая точка алгоритма обучения достигнет окрестности глобального минимума. Соответственно, возникает необходимость остановить алгоритм обучения сразу после достижения максимума обобщающей способности ИНС. Признаком того, что рабочая точка алгоритма достигла окрестности глобального минимума, является малое изменение абсолютной ошибки в течение эпохи обучения:

$$
\frac{1}{C} \sum_{j \in C} \Delta e_{j}(n) \leq 0.005-0.01,
$$

где $\Delta e_{j}(n)-$ изменение абсолютной ошибки нейрона $j$ выходного слоя в течение эпохи обучения $n$.

Модели прогнозирования электропотребления могут быть классифицированы на две категории: одиночные модели прогнозирования и ансамбли из нескольких моделей [8]. Использование композиционных методов является одним из самых действенных подходов к решению задачи классификации образов. Композиционные методы (или ансамбли) классификации формируют набор различных моделей классификации для достижения лучшей точности, чем у каждой модели в отдельности [31]. Ансамбль представляет собой ассоциативную машину, состоящую из нескольких параллельно работающих ИНС. Отклики каждой нейронной сети, входящей в ансамбль, некоторым образом комбинируются, формируя итоговый отклик ассоциативной машины. Использование ансамблей нейронных сетей при STLF рассмотрено в $[8,32]$. Особо важное значение имеет способ получения итогового отклика ассоциативной машины. В настоящее время хорошо зарекомендовали себя ансамбли ИНС одинаковой архитектуры с различной начальной конфигурацией, которые начинают обучение из различных положений на поверхности ошибки. Для получения итогового выходного сигнала используется блок усреднения по ансамблю.

\section{Прогнозирование почасовых объемов электропотребления ГТП ГП второго уровня с помощью многослойного персептрона}

Прогнозирование потребления электроэнергии является сложной задачей, так как оно зависит от сложного поведения электрической нагрузки зданий (сооружений) и неопределенности влияющих факторов, 
что, в свою очередь, приводит к частым колебаниям спроса на электроэнергию. Эти колебания обусловлены архитектурой зданий и тепловыми свойствами используемых материалов, поведением жителей, климатическими условиями и подуровнем системной компоненты, таким как освещение или HVAC (отопление, вентиляция, и кондиционирование) [33]. Прогнозирование почасового электропотребления ГТП гарантирующего поставщика второго уровня с помощью инструментов нейросетевого анализа начинается с подготовки выборки, необходимой для обучения и тестирования обобщающей способности ИНС. Для формирования обучающей выборки используются статистические данные о фактическом потреблении ГТП и величинах всех влияющих факторов с 01.04.2016. Использование более ранних статистических данных ухудшает результаты прогнозирования вследствие того, что 27.03.2016 произошел последний переход на летнее время (существенно изменилась форма суточных графиков потребления электроэнергии). Далее база статистических данных о фактических величинах влияющих факторов кодируется для удобства представления ИНС. Наконец, статистический ряд данных об электрической нагрузке и величине основных влияющих факторов должен быть нормализован к диапазону [0; 1] [34], который соответствует области определения сигмоидальной функции активации. Также для ускорения сходимости алгоритма необходимо сдвинуть границы диапазона нормализации из зоны насыщения логистической функции вблизи границ области определения:

$$
x_{\text {норм }}=\frac{x-x_{\min }}{x_{\max }-x_{\min }} \cdot 0,9+0,05,
$$

где $x_{\text {норм }}$ - нормализованное значение входных данных; $x$ - фактическое значение входных данных; $x_{\text {min }}$, $x_{\max }$ - минимальное и максимальное значения ряда входных данных. В табл. 1 представлен фрагмент нормализованных данных обучающей выборки. Нормализация данных производится на основании выражения (11).

Один обучающий пример (training sample) представляет собой массив $13 \times 24$ элемента, соответствующий суточным значениям всех влияющих факторов из табл. 1. Перед подачей на вход ИНС данный массив преобразуется в одномерный вектор-столбец $1 \times 312$ элементов. Желаемый отклик ИНС представляет собой вектор-столбец размером 1×24 элемента, соответствующий суточным значениям электропотребления ГТП (столбец 3, табл. 1).

Массив обучающих данных произвольным образом делится на обучающую и тестовые выборки в соотношении 9:1. Для более точной оценки обобщающей способности ИНС данные тестовой выборки должны изменяться после каждой итерации обучающего цикла.

Практическая реализация прогнозной модели осуществлена с помощью библиотеки глубокого машинного обучения Tensorflow Keras на языке программирования Python 3.6. Построен ансамбль, состоящий из трех четырехслойных персептронов, обучение которых происходит с помощью алгоритма ADAM. В соответствии с выражениями (4), (5), размер входного слоя каждой ИНС, входящей в ассоциативную машину, $m_{0}=312$, размер первого скрытого слоя $m_{1}=139$, размер второго скрытого слоя $m_{2}=58$, размер выходного слоя $m_{3}=24$. Для получения итогового отклика ассоциативной машины используется блок усреднения по ансамблю.

Обучение ИНС происходит в последовательном режиме, когда свободные параметры ИНС корректируются после подачи каждого примера из обучающей выборки. Максимальное количество эпох обучающего цикла составляет 100. Инструментарий Tensorflow Keras позволяет реализовать функцию ранней остановки алгоритма обучения для борьбы с переобучением ИНС. Критерием остановки является выполнение условия выражения (10) в течение пяти итераций обучающего цикла.

После окончания обучения свободные параметры ИНС «замораживаются». Для получения прогноза электропотребления на следующие сутки необходимо произвести опрос ИНС примером данных, соответствующих суткам, на которые осуществляется прогнозирование. В табл. 2 представлены результаты почасового прогноза электропотребления ГТП «Власиха» на следующие сутки в период 1-30 июня 2019.

Таблица 1. Фрагмент нормализованных данных обучающей выборки

Table 1. Detail of normalized training data

\begin{tabular}{|c|c|c|c|c|c|c|c|c|c|c|c|c|}
\hline 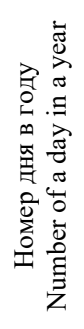 & 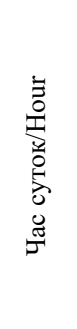 & 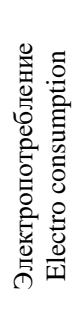 & 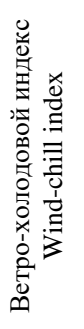 & 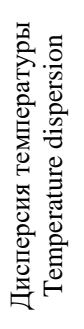 & 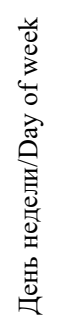 & 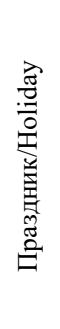 & 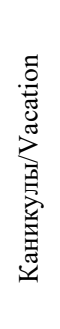 & 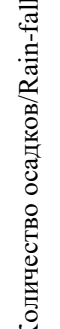 & 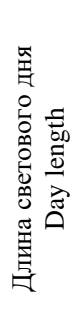 & 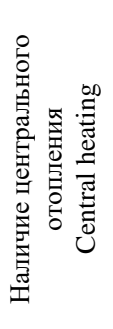 & 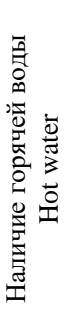 & 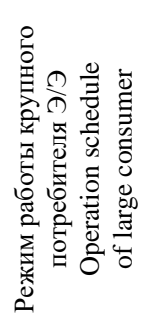 \\
\hline \multicolumn{13}{|c|}{ Относительные единицы/Rel. units } \\
\hline 0,277 & 0,05 & 0,19 & 0,591 & 0,104 & 0,95 & 0,35 & 0,95 & 0,05 & 0,581 & 0,95 & 0,95 & 0,95 \\
\hline 0,277 & 0,089 & 0,204 & 0,581 & 0,103 & 0,95 & 0,35 & 0,95 & 0,05 & 0,581 & 0,95 & 0,95 & 0,95 \\
\hline
\end{tabular}


Таблица 2. Результаты почасового прогноза электропотребления группы точек поставки электроэнергии «Власиха» на следующие сутки в период 1-30 июня 2019

Table 2. Result of hourly load forecasting for the next day of delivery point cluster "Vlasikha» in period 1-30 June 2019

\begin{tabular}{|c|c|c|}
\hline $\begin{array}{c}\text { Месяц, год } \\
\text { Month, year }\end{array}$ & $\begin{array}{c}\text { Ошибка метода } \\
\text { экспертных оценок } \\
\text { Error of Delhi approach, \% }\end{array}$ & $\begin{array}{c}\text { Ошибка ансамбля } \\
\text { нейронных сетей } \\
\text { Error of ensemble } \\
\text { neural network, \% }\end{array}$ \\
\hline $\begin{array}{c}\text { Июнь 2019 } \\
\text { June 2019 }\end{array}$ & 3,07 & 2,92 \\
\hline
\end{tabular}

\section{Заключение}

В исследовании произведен анализ факторов, влияющих на поведение временного ряда электропотребления. Выяснено, что поведение временного ряда электропотребления группы точек поставки электроэнергии в краткосрочной перспективе в основном определяют временные и метеорологические факторы. Для группы точек поставки электроэнергии гарантирующих поставщиков второго уровня также необхо-

\section{СПИСОК ЛИТЕРАТУРЫ}

1. Бояркин Д.А., Крупенев Д.С., Якубовский Д.В. Использование методов машинного обучения при оценке надежноти электроэнергетических систем методом Монте-Карло // Вестник ЮУрГУ. Серия «Математическое моделирование и программирование». - 2018. - Т. 11. - № 4. - С. 146-153.

2. Empirical comparison of neural network and auto-regressive models in short-term load forecasting / M. López, C. Sans, S. Valero, C, Senabre // Energies. - 2018. - V. 11. - P. 1-19. DOI: 10.3390/en11082080.

3. Tureczek A., Nielsen P., Madsen H. Electricity consumption clustering using smart meter data // Energies. - 2018. - V. 11. P. 1-18. DOI: 10.3390/en11040859.

4. Zheng H., Yuan J., Chen L. Short-term load forecasting using EMD-LSTM neural networks with a XGBOOSt algorithm for feature importance evaluation // Energies. - 2017. - V. 10. - P. 1-20. DOI: $10.3390 /$ en10081168.

5. Katasev A.S. Neuro-fuzzy model of fuzzy rules formation for objects state evaluation in conditions of uncertainty // Computer research and modeling. -2019 . - V. 11. - № 3. - P. 477-492. DOI 10.20537/2076-7633-2019-11-3-477-492.

6. Иванин О.А., Директор Л.Б. Применение искусственных нейронных сетей для прогнозирования энергетических нагрузок обособленных потребителей // Теплоэнергетика. - 2018. № 5. - C. 17-26. DOI: 10.1134/S0040363618050041.

7. Optimal deep learning LSTM model for electric load forecasting using feature selection and genetic algorithm: comparison with machine learning approaches / S. Bouktif, A. Fiaz, A. Ouni, M. Serhani // Energies. - 2018. - V. 11. - P. 1-20. DOI: 10.3390/en11071636.

8. Wang Z., Srinivasan R. A review of artificial intelligence based building energy use prediction: contrasting the capabilities of single and ensemble prediction models // Renewable and Sustainable Energy Reviews. - 2017. - V. 75. - P. 796-808.

9. Operational supply and demand optimisation of a multi-vector district energy system using artificial neural networks and a genetic algorithm / J. Reynolds, M.W. Ahmad, Y. Rezgui, J. Hippolyte // Applied Energy. - 2019. - V. 235. - P. 699-713.

10. Hybridizing chaotic and quantum mechanisms and fruit fly optimization algorithm with least square ssupport vector regression model in electric load forecasting / M.W. Li, J. Geng, W.C. Hong, Y. Zhang // Energies. - 2018. - V. 11. - P. 1-22. DOI 10.3390/en11092226.

11. Грачев В.И., Губин С.П. Научно-технологическое прогнозирование // Радиоэлектроника. Наносистемы. Информационные технологии. - 2017. - Т. 9. - № 1. - С. 3-4. димо учитывать факторы надежности питающей электросети и режим работы крупных потребителей электроэнергии группы точек поставки электроэнергии, а также наличие центрального отопления и горячего (холодного) водоснабжения в населенных пунктах.

На основе исходной статистической информации разработан нейросетевой алгоритм краткосрочного прогнозирования электропотребления групп точек поставки электроэнергии гарантирующего поставщика второго уровня. Для улучшений сходимости алгоритма обучения используются адаптивные в процессе обучения параметры скорости обучения и момента инерции. Программная реализация данного алгоритма выполнена с помощью библиотеки глубокого машинного обучения Tensor flow Keras на языке программирования Python 3.6. Использование данной искусственной нейронной сети позволило снизить, по сравнению с методом экспертных оценок, среднемесячную относительную ошибку прогнозирования на $5,14 \%$.

12. Scalable clustering of individual electrical curves for profiling and bottom-up forecasting / B. Auder, J. Cugliari, Y. Goude, J. Poggi // Energies. - 2018. - V. 11. - P. 1-22. DOI: 10.3390/en11071893.

13. Rajan D.V., Sourav M., Thakur S.S. An efficient approach for short-term load forecasting using historical data // International Journal of Engineering Research \& Technology. - 2012. - V. 3. P. 1-9.

14. Ruzic S., Vuckovic A., Nikolic N. Weather sensitive method for short term load forecasting in electric power Utility of Serbia // IEEE Transactions on Power Systems. - 2003. - V. 18. - № 4. P. 1581-1586.

15. Quayle R.G., Steadman R.G. The Steadman wind chill: an improvement over present scales // Amer. Meteorological Soc. 1998. - V. 13. -P. 1187-1193.

16. Карамов Д.Н., Наумов И.В., Пержабинский С.М. Математическое моделирование отказов элементов электрической сети (10 кв) автономных энергетических систем с возобновляемой распределенной генерацией // Известия Томского политехнического университета. Инжиниринг георесурсов. - 2018. T. 329. - № 7. - C. 116-130.

17. Третьяк А.Я., Кузнецова А.В., Борисов К.А. Определение поломок резцов $\mathrm{PCD}$ с помощью регрессионного и нейросетевого моделирования // Известия Томского политехнического университета. Инжиниринг георесурсов. - 2019. - Т. 330. № 5. - C. 169-177.

18. A review of data-driven approaches for prediction and classification of building energy consumption / Y. Weia, X. Zhangb, Y. Shia, et al. // Renewable and Sustainable Energy Reviews. - 2018. - V. 82. - P. 1027-1047.

19. Hayes A. First links in the Markov chain // American Scientist. 2013. - V. 101. - P. 92-97.

20. Prokoptsev N.G., Alekseenko A.E., Kholodov Ya.A. Traffic flow speed prediction on transportation graph with convolutional neural networks // Computer research and modeling. - 2018. - V. 10. № 3. - P. 359-367. DOI: 10.20537/2076-7633-2018-10-3-359-367.

21. Timofeeva G.A. Forecasting the return of the loan portfolio on the basis of Markov model // Bulletin of the South Ural State University. Ser. Mathematical Modelling, Programming \& Computer Software (Bulletin SUSU MMCS). - 2017. - V. 10. № 3. - P. 54-66. DOI: 10.14529/mmp170305.

22. Августинович В.Г., Кузнецова Т.А., Нугуманов А.Д. Разработка нейронных систем мониторинга и управления эмиссией вредных веществ для газотурбинных газоперекачивающих агрегатов и электростанций // Известия Томского политехнического университета. Инжиниринг георесурсов. - 2019. - Т. 330. № 8. - C. 7-17. DOI: 10.18799/24131830/2019/8/2207. 
23. Воевода А.А., Романников Д.О. Синтез нейронной сети для реализации рекуррентного метода наименьших квадратов // Научный вестник НГТУ. - 2018. - № 3 (72). - С. 33-42. DOI: 10.17212/1814-1196-2018-3-33-42.

24. Genco S., Yildiray P., Umran S. An artificial neural network model for $\mathrm{Na} / \mathrm{K}$ geothermometer // Proceedings World Geothermal Congress. - Bali, 2010. - P. 1-10.

25. Ryu S., Noh J., Kim H. Deep neural network based demand side short term load forecasting // Energies. - 2017. - V. 10. - № 3. P. 1-20. DOI: 10.3390/en10010003

26. Использование нейро-нечетких диагностических моделей при оценке технического состояния электрооборудования тепловоза / А.В. Агунов, А.В. Грищенко, В.А. Крючек, В.В. Грачев // Электротехника. - 2017. - № 10. - С. 14-17.

27. Alkousa M.S. On some stochastic mirror descent methods for constrained online optimization problems // Computer research and modeling. - 2019. - V. 11. - № 2. - P. 205-217. DOI 10.20537/2076-7633-2019-11-2-205-217

28. Kingma D.P., Lei Ba J. ADAM: a method for stochastic optimization // ArXiv.org. 2015. URL: https://arxiv.org/abs/1412.6980 (дата обращения 12.12.2019)

29. Rahmana A., Srikumarb V., Smith A. Predicting electricity consumption for commercial and residential buildings using deep recurrent neural networks // Applied Energy. - 2018. - V. 212. P. 372-385.

30. Dropout: a simple way to prevent neural networks from overfitting / N. Srivastava, G. Hinton, A. Krizhevsky, I. Sutskever, R. Salakhutdinov // Journal of Machine Learning Research. 2014. - V. 15. - P. 1929-1958.

31. Жуков А.В., Сидоров Д.Н. Модификация алгоритма случайного леса для классификации нестационарных потоковых данных // Вестник ЮУрГУ. Серия «Математическое моделирование и программирование». - 2016. - Т. 9. - № 4. - С. 86-95. DOI: $10.14529 / \mathrm{mmp} 160408$.

32. Li S., Wang P., Goel L. A novel wavelet-based ensemble method for short-term load forecasting with hybrid neural networks and feature selection // IEEE Transactions on Power Systems. 2016. - V. 31. - № 3. - P. 1788-1798.

33. Deep learning for estimating building energy consumption / E. Mocanu, P.H. Nguyen, M. Gibescu, W.L. Kling // Sustainable Energy, Grids and Networks. - 2016. - V. 6. - P. 91-99.

34. A hybrid forecasting model with parameter optimization for shortterm load forecasting of micro-grids / N. Liu, Q. Tang, J. Zhang, W. Fan, J. Liu // Applied Energy. - 2014. - V. 129. - P. 336-345.

Поступила 03.06.2020 г.

\section{Информация об авторах}

Хомутов C.O., доктор технических наук, профессор кафедры электроснабжения промышленных предприятий Алтайского государственного технического университета им. И.И. Ползунова.

Сташко В.И., кандидат технических наук, доцент кафедры электроснабжения промышленных предприятий Алтайского государственного технического университета им. И.И. Ползунова.

Серебряков Н.А., ассистент кафедры электроснабжения промышленных предприятий Алтайского государственного технического университета им. И.И. Ползунова. 
UDC 621.316 .72

\title{
IMPROVING THE ACCURACY OF SHORT-TERM LOAD FORECASTING OF DELIVERY POINT CLUSTER OF THE SECOND LEVEL DEFAULT PROVIDER
}

\author{
Stanislav 0. Khomutov 1 , \\ homutov.so@yandex.ru \\ Vasiliy I. Stashko ${ }^{1}$, \\ stashko@list.ru \\ Nikolay A. Serebryakov ${ }^{1}$, \\ na_serebryakov@altke.ru \\ 1 Polzunov Altai State Technical University, \\ 46, Lenin avenue, Barnaul, 656038, Russia.
}

Relevance of the discussed issue is caused by the need to improve the accuracy of short-term load forecasting of delivery point cluster of the second level default provider. The system operator uses the result of forecast when forming power system dispatch load curve. Usually, prediction errors lead to increase of primary energy resources consumption for electric-power production, due to unjustified run and shutdown of generating equipment, as well as increasing of circuit losses, due to the choice of non-optimal scheme of electric grid. As the electricity consumption depends on many factors, the task of short-term load forecasting is poorly formalized. Under these conditions, traditional methods of mathematical statistics and simulation do not allow building the adequate forecast models. Until recently, the only fine method of load forecasting was the Delhi approach. Currently, tools of neural networks and deep machine learning are widely used for short-term load forecasting of the energy system of a region of the country or delivery point cluster of first level default provider. However, the developed models are not suitable for predicting hourly electricity consumption of delivery point cluster of the second level default provider. Short-term load forecasting of this object is complicated of reliability of electric grid 6-110 kV, the operating mode of electricity consumers with a capacity of 670-10000 kW, the presence of district heating and water supply, beside standard time and meteorological factors. For this forecasting object, the question of choosing the optimal architecture and configuration of the neural network model, as well as the learning algorithm, which can achieve the desired forecasting accuracy, remain open.

The main aim of the research is to improve the accuracy of short-term load forecasting of delivery point cluster of the second level default provider with the help of tools of neural networks and deep machine learning.

The methods: the methods of correlation and factor analysis, the theory of artificial neural networks and machine learning. Software implementation of theoretical calculations was performed with help of deep machine learning library Tensor flow Keras in the Python 3.6 programming language.

Results. The authors have developed the neural network algorithm for short-term load forecasting of delivery point cluster of the second level default provider with adaptive learning and momentum rate and completed the software implementation of this algorithm in deep machine learning library Tensor flow Keras. The use of this artificial neural network let to decrease in monthly average relative forecast error by $5,14 \%$.

\section{Key words:}

Short-term load forecasting, artificial neural networks, learning algorithm, wholesale electricity market, delivery point cluster, gradient descent.

\section{REFERENCES}

1. Boyarkin D.A., Krupenev D.S., Iakubovskii D.V. Machine learning in electric power systems adequacy assessment using Monte Carlo method. Bulletin of the South Ural StateUniversity. Ser. Mathematical Modelling, Programming \& Computer Software (Bulletin SUSU MMCS), 2018, vol. 11, no. 4, pp. 146153. In Rus.

2. López M., Sans C., Valero S., Senabre C. Empirical comparison of neural network and auto-regressive models in short-term load forecasting. Energies, 2018, vol. 11, pp. 1-19. DOI: 10.3390/en11082080.

3. Tureczek A., Nielsen P., Madsen H. Electricity consumption clustering using smart meter data. Energies, 2018, vol. 11, pp. 118. DOI: $10.3390 /$ en11040859.

4. Zheng H., Yuan J., Chen L. Short-term load forecasting using EMD-LSTM neural networks with a XGBOOSt algorithm for feature importance evaluation. Energies, 2017, vol. 10, pp. 1-20. DOI: $10.3390 /$ en10081168.

5. Katasev A.S. Neuro-fuzzy model of fuzzy rules formation for objects state evaluation in conditions of uncertainty. Computer research and modeling, 2019, vol. 11, no. 3 pp. 477-492. DOI: 10.20537/2076-7633-2019-11-3-477-492.
6. Ivanin O. A., Direktor L. B. Application of artificial neural networks to predict energy loads of isolated consumers. Thermal Engineering, 2018, no. 5, pp. 17-26. DOI: 10.1134/S0040363618050041. In Rus.

7. Bouktif S., Fiaz A., Ouni A., Serhani M. Optimal deep learning LSTM model for electric load forecasting using feature selection and genetic algorithm: comparison with machine learning approaches. Energies, 2018, vol. 11, pp. 1-20. DOI: 10.3390/en11071636.

8. Wang Z., Srinivasan R. A review of artificial intelligence based building energy use prediction: Contrasting the capabilities of single and ensemble prediction models. Renewable and Sustainable Energy Reviews, 2017, vol. 75, pp. 796-808.

9. Reynolds J., Ahmad M.W., Rezgui Y., Hippolyte J. Operational supply and demand optimisation of a multi-vector district energy system using artificial neural networks and a genetic algorithm. Applied Energy, 2019, vol. 235, pp. 699-713.

10. Li M.W., Geng J., Hong W.C., Zhang Y. Hybridizing chaotic and quantum mechanisms and fruit fly optimization algorithm with least square ssupport vector regression model in electric load forecasting. Energies, 2018, vol. 11, vol. 2226, pp. 1-22. DOI: 10.3390/en11092226.

11. Grachev V.I., Gubin P.S. Scientific-technological forecasting. Radioelectronics. Nanosystems. Information Technologies, 2017, vol. 9, no. 1, pp. 3-4. In Rus. DOI: 10.17725/rensit.2017.09.003. 
12. Auder B., Cugliari J., Goude Y., Poggi J. Scalable clustering of individual electrical curves for profiling and bottom-up forecasting Energies, 2018, vol. 11, pp. 1-22. DOI: 10.3390/en11071893.

13. Rajan D.V., Sourav M., Thakur S.S. An efficient approach for short-term load forecasting using historical data. International Journal of Engineering Research \& Technology, 2012, vol. 3, pp. $1-9$.

14. Ruzic S., Vuckovic A., Nikolic N. Weather sensitive method for short term load forecasting in electric power Utility of Serbia. IEEE Transactions on Power Systems, 2003, vol. 18, no. 4, pp. 1581-1586.

15. Quayle R.G., Steadman R.G. The Steadman wind chill: an improvement over present scales. Amer. Meteorological Soc., 1998. vol. 13. pp. $1187-1193$

16. Karamov D.N., Naumov I.V., Perzhabinsky S.M. Mathematical modelling of failures of electrical grid $(10 \mathrm{kV})$ of autonomous energy systems with renewable distributed generation. Bulletin of the Tomsk Polytechnic University. Geo Assets Engineering, 2018, vol. 329, no. 7, pp. 116-130. In Rus.

17. Tretyak A.Y., Kuznetsova A.V., Borisov K.A. Determination of PDC cutter breakdowns using regression and neural network modeling. Bulletin of the Tomsk Polytechnic University. Geo Assets Engineering, 2019, vol. 330, no. 5, pp. 169-177. In Rus.

18. Wei Y., Zhangb X., Shia Y. A review of data-driven approaches for prediction and classification of building energy consumption. Renewable and Sustainable Energy Reviews, 2018, vol. 82, pp. 1027-1047.

19. Hayes A. First links in the Markov chain. American Scientist, 2013 vol. 101, pp. 92-97.

20. Prokoptsev N.G., Alekseenko A.E., Kholodov Ya.A. Traffic flow speed prediction on transportation graph with convolutional neural networks. Computer research and modeling, 2018, vol. 10, no. 3 , pp. 359-367. DOI: 10.20537/2076-7633-2018-10-3-359-367.

21. Timofeeva G.A. Forecasting the return of the loan portfolio on the basis of Markov model. Bulletin of the South Ural State University. Ser. Mathematical Modelling, Programming \& Computer Software (Bulletin SUSU MMCS), 2017, vol. 10, no. 3, pp. 54-66. DOI: $10.14529 / \mathrm{mmp} 170305$.

22. Avgustinovich V.G., Kuznetsova T.A., Nugumanov A.D Development of neural systems for monitoring and controlling emission of gas-transmission and power gas turbine units. Bulletin of the Tomsk Polytechnic University. Geo Assets Engineering, 2019, vol. 330, no. 8, pp. 7-17. DOI: $10.18799 / 24131830 / 2019 / 8 / 2207$. In Rus.

23. Voevoda A.A., Romannikov D.O. Synthesis of a neural network for the implementation of the recursive leastsquares method.
Science bulletin of the Novosibirsk state technical university, 2018 , no. 3 (72), pp. 33-42. In Rus. DOI: 10.17212/1814-1196-2018-3-33-42.

24. Genco S., Yildiray P., Umran S. An artificial neural network model for Na/K geothermometer. Proceedings World Geothermal Congress. Bali, 2010. pp. 1-10.

25. Ryu S., Noh J., Kim H. Deep neural network based demand side short term load forecasting. Energies, 2017, vol. 10, no. 3, pp. 120. DOI: 10.3390/en 10010003

26. Agunov A.V., Grishchenko A.V., Kruchek V.A., Grachev V.V. A method of using neural fuzzy models to determine the technical state of a diesel locomotive's electrical equipment. Russian Electrical Engineering, 2017, vol. 88, no. 10, pp. 634-638. In Rus.

27. Alkousa M.S. On some stochastic mirror descent methods for constrained online optimization problems. Computer research and modeling, 2019, vol. 11, no. 2, pp. 205-217. DOI: 10.20537/20767633-2019-11-2-205-217.

28. Kingma D.P., Lei Ba J. ADAM: a method for stochastic optimization. ArXiv.org, 2015. Available at: https://arxiv.org/abs/1412.6980 (accessed 12 December 2019).

29. Rahmana A., Srikumarb V., Smith A. Predicting electricity consumption for commercial and residential buildings using deep recurrent neural networks. Applied Energy, 2018, vol. 212, pp. 372385.

30. Srivastava N., Hinton G., Krizhevsky A., Sutskever I., Salakhutdinov R. Dropout: a simple way to prevent neural networks from overfitting. Journal of Machine Learning Research, 2014, vol. 15, pp. 1929-1958.

31. Zhukov A.V., Sidorov D.N. Modification of random forest based approach for streaming data with concept drift. Bulletin of the South Ural State University. Ser. Mathematical Modelling, Programming \& Computer Software (Bulletin SUSU MMCS), 2016, vol. 9, no. 4, pp. 86-95. DOI: 10.14529/mmp160408. In Russ.

32. Li S., Wang P., Goel L. A novel wavelet-based ensemble method for short-term load forecasting with hybrid neural networks and feature selection. IEEE Transactions on Power Systems, 2016, vol. 31, no. 3, pp. 1788-1798.

33. Mocanu E., Nguyen P.H., Gibescu M., Kling W.L. Deep learning for estimating building energy consumption. Sustainable Energy, Grids and Networks, 2016, vol. 6, pp. 91-99.

34. Liu N., Tang Q., Zhang J., Fan W., Liu J. A hybrid forecasting model with parameter optimization for short-term load forecasting of micro-grids. Applied Energy, 2014, vol. 129, pp. 336-345.

Received: 3 June 2020.

\section{Information about the authors}

Stanislav O. Khomutov, Dr. Sc., professor, Polzunov Altai State Technical University.

Vasiliy I. Stashko, Cand. Sc., associate professor, Polzunov Altai State Technical University.

Nikolay A. Serebryakov, assistant, Polzunov Altai State Technical University. 